\title{
COMPOSITION DU COMITÉ RST
DE L'ACADÉMIE DES SCIENCES
}

\section{Christian AMATORE}

Membre de l'Académie des sciences - Directeur de recherche au Centre national de la recherche et à l'École normale supérieure

\section{François BACCELLI}

Membre de l'Académie des sciences - Directeur de recherche à l'Institut national de recherche en informatique et en automatique

\section{Jean-François BACH}

Secrétaire perpétuel de l'Académie des sciences - Professeur à l'université RenéDescartes

\section{Roger BALIAN}

Membre de l'Académie des sciences - Physicien à l'Institut de physique théorique - CEA Saclay

\section{Alain CARPENTIER}

Vice-président de l'Académie des sciences - Professeur émérite à l'université Pierre-et-Marie-Curie

\section{Patrick CHARNAY}

Membre de l'Académie des sciences - Directeur de recherche à l'Institut national de la santé et de la recherche médicale

\section{François CUZIN}

Membre de l'Académie des sciences - Professeur à l'université de Nice-SophiaAntipolis

\section{Michel DAVIER}

Membre de l'Académie des sciences - Professeur à l'université Paris-Sud Orsay

\section{Henri DÉCAMPS}

Membre de l'Académie des sciences - Directeur de recherche émérite au Centre national de la recherche scientifique 


\section{Jean DERCOURT}

Secrétaire perpétuel de l'Académie des sciences - Professeur émérite à l'université Pierre-et-Marie-Curie

\section{Christian DUMAS}

Membre de l'Académie des sciences - Professeur à l'École normale supérieure de Lyon

\section{Pierre ENCRENAZ}

Membre de l'Académie des sciences - Professeur à l'université Pierre-et-MarieCurie

\section{Marc JEANNEROD}

Membre de l'Académie des sciences - Professeur émérite à l'université ClaudeBernard

\section{Jean-Pierre KAHANE}

Membre de l'Académie des sciences - Professeur émérite à l'université Paris-Sud Orsay

\section{Bernard MEUNIER}

Membre de l'Académie des sciences - Président-directeur général de Palumed

\section{Paul-Henri REBUT}

Correspondant de l'Académie des sciences - Ancien directeur du Jet et d'Iter Conseiller d'Iter

\section{Jean SALENCCON}

Président de l'Académie des sciences - Membre de l'Académie des technologies - Ingénieur général honoraire des ponts et chaussées - Professeur honoraire à l'École polytechnique et à l'École nationale des ponts et chaussées

\section{Erich SPITZ}

Correspondant de l'Académie des sciences - Membre de l'Académie des technologies - Conseiller du groupe Thales

\section{Pierre SUQUET}

Membre de l'Académie des sciences - Directeur de recherche au Centre national de la recherche scientifique

\section{Philippe TAQUET}

Membre de l'Académie des sciences - Professeur au Muséum national d'histoire naturelle

\section{Alain-Jacques VALLERON}

Membre de l'Académie des sciences - Professeur à l'université Pierre-et-MarieCurie 


\section{Jean-Christophe YOCCOZ}

Membre de l'Académie des sciences - Professeur au Collège de France

\section{Coordination éditoriale :}

\section{Joëlle FANON}

Adjointe du directeur du service des publications de l'Académie des sciences 


\section{KVVDJHIQQMQWRQDQIOHWEDQN}

